\title{
Preference for different visual stimulus sequences in children, adolescents, and young adults
}

PAUL B. BALTES, ${ }^{2}$ DEPARTMENT OF PSYCHOLOGY. WEST VIRGINIA UNIVERSITY, Morgantown, W. Va. 26506 AND LOTHAR R. SCHMIDT AND ERNEST E. BOESCH, UNIVERSITAET DES SAARLANDES, Germany

Using a cross-sectional approach male $S$ s ranging in age from 8 to 20 years were asked to make preference judgments with regard to all possible pairs of eight different visual stimulus sequences. The stimulus sequences consisted of a different number $(3,5,8$, $13,22,37,61,1.00)$ of single light flashes spaced equally within a $10 \mathrm{sec}$ interval. With increasing age Ss preferred slower stimulus sequences. These results are in close agreement with earlier results on auditory stimulus material.

Starting from the work of Fiske \& Maddi (1961), Leuba (1962), and Berlyne (1960), the present authors have recently presented data (Boesch, Baltes, \& Schmidt, 1968) indicating marked age-differences in preferences for auditory stimulus sequences differing in number of single tone units spaced equally within a $10 \mathrm{sec}$ interval. With increasing age, between 8 and 20 years, Ss preferred stimulus sequences containing fewer tone units, i.e., slower stimulus sequences. The present study investigates, whether the same pattern of preferences can be replicated when visual rather than auditory stimulus material is used.

Subjects and Stimuli. Using a cross-sectional approach, six comparable male samples with mean ages of about $8(\mathrm{~N}=29), 10(\mathrm{~N}=29), 12(\mathrm{~N}=28)$, $14(\mathrm{~N}=32), 17(\mathrm{~N}=37)$, and $20(\mathrm{~N}=27)$ years participated in the experiment. These were the same Ss that were used in the study on preferences for different auditory stimulus sequences (Boesch et al, 1968). Visual and auditory stimulus sequences had been given in one session in counterbalanced order. There was, however, no evidence for carry-over effects of visual on auditory or of auditory on visual presentation.

The visual stimulus sequences were constructed in analogy to the auditory

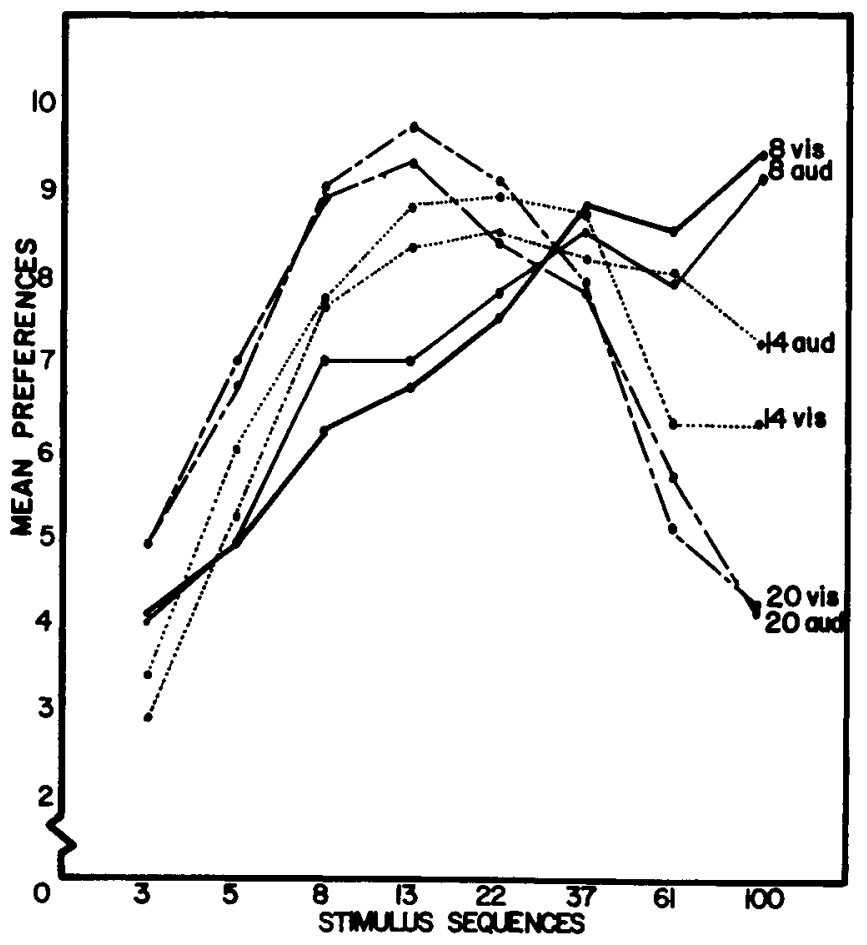

Fig. 1. Mean preferences of three age groups $(8,14,20)$ for eight visual (vis) and auditory (aud) stimulus sequences.
Table 1

Mean Preferences for Stimulus Sequences (SS) Six Age Groups

\begin{tabular}{rcccccccr} 
Age/SS & 3 & 5 & 8 & 13 & 22 & 37 & 61 & 100 \\
\hline 8 & 4.1 & 4.9 & 6.2 & 6.7 & 7.5 & 8.8 & 8.5 & 9.4 \\
10 & 3.3 & 4.5 & 5.9 & 6.8 & 7.9 & 8.3 & 9.0 & 10.3 \\
12 & 3.3 & 5.3 & 7.3 & 9.1 & 8.7 & 8.7 & 6.8 & 6.9 \\
14 & 3.4 & 6.0 & 7.7 & 8.8 & 8.9 & 8.7 & 6.3 & 6.3 \\
17 & 3.1 & 5.5 & 7.7 & 8.9 & 9.4 & 9.0 & 6.7 & 5.8 \\
20 & 4.9 & 6.7 & 9.0 & 9.7 & 8.6 & 7.9 & 5.1 & 4.2 \\
\hline
\end{tabular}

stimulus material. A total of eight visual stimulus sequences were used. The sequences differed only in the number of single light flashes spaced equally within a $10 \mathrm{sec}$ interval. The number of single light flashes was chosen to vary in logarithmic steps from 3 to 100 per 10 sec interval, i.e., $3,5,8,13,22,37$, 61 , and 100. Each single light flash had an intensity of about $15 \mathrm{~W}$, a duration of $50 \mathrm{msec}$, and was of red-yellowish color. It was produced by six bulbs which were covered by a round diffusion screen $10 \mathrm{~cm}$ in diameter.

Groups of five Ss each were presented in a complete pair-comparison design with all 28 possible pairs of the eight visual stimulus sequences. The order of presentation being defined by chance was the same as that for the auditory sequences. The two stimulus sequences comprising each pair were given successively with a pause of 3 sec distinguishing them. The interval between each of the 28 pairs was $10 \mathrm{sec}$. During this latter pause, the Ss were asked to indicate on a mimeographed sheet which one of the two visual stimulus sequences they "liked best." After one week all Ss were re-tested under the same procedure.

Results and Discussion. Each $\mathrm{S}$ received a score based on the number of preferences he stated for each stimulus sequence on both sessions, so that each $S$ had a total of 56 preferences distributed over the eight stimulus sequences. The mean preferences for the stimulus sequences across the six age groups are presented in Table 1.

After the over-all age by stimulus sequence interaction was found to be significant at the $1 \%$ level of confidence, separate one-way analyses of variance were performed for each of the eight stimulus sequences with the single factor being age in six levels. These analyses of variance showed significant age differences for five of the eight stimulus sequences $(8,13,22,61$, and 100) at the $1 \%$ level of confidence. As can be seen from Table 1 , these age differences signify that with regard to the slower stimulus sequences $(8,13,22)$ the older Ss received higher preference scores, while the younger age groups showed higher preference scores for the faster stimulus sequences $(61,100)$. Over-all, the results can be described as Ss prefer with increasing age slower visual stimulus sequences with a marked age development around 10-12 years of age. The stimulus sequence with the highest preference score is, e.g., 100 in the 8 year-, 22 in the 14 year-, and 13 in the 20-year-olds.

The age trend towards preference for slower auditory stimulus sequences (Boesch et al, 1968) seems to hold also for visual stimulus material. Using auditory stimuli significant age differences had been found for the sequences $5,8,13,61$, and 100 , while the present study, using visual material, yielded age differences for the stimulus sequences $8,13,22,61$, and 100 . The similarity in results for visual and auditory sequences is illustrated in Fig. 1 which shows the mean preferences for the 8-, 14, and 20-year-olds.

More systematic evidence on the relation between preferences for auditory and visual stimulus material was obtained by computing rank correlations of mean preferences between age groups both within and across sensory modalities. First, agecorrelations were computed separately for within auditory and for within visual stimulus sequences. Both age-correlation matrices 
Table 2

Correlations Between Age Groups Comparing Mean Preferences Across Auditory and Visual Stimulus Sequences (SS)

\begin{tabular}{rllllll} 
Age/Age & 8 & 10 & 12 & 14 & 17 & 20 \\
\hline 8 & $99 * *$ & $97^{* *}$ & 38 & 41 & 45 & -20 \\
10 & $93^{* *}$ & $98^{* *}$ & 31 & 33 & 40 & -19 \\
12 & $73^{*}$ & $64^{*}$ & $85^{* *}$ & $85^{* *}$ & $86^{* *}$ & 41 \\
14 & 45 & 43 & $90^{* *}$ & $93^{* *}$ & $95^{* *}$ & $67^{*}$ \\
17 & 38 & 33 & $81^{*}$ & $86^{* *}$ & $95^{* *}$ & $67^{*}$ \\
20 & -19 & -24 & $76^{*}$ & $71^{*}$ & $69^{*}$ & $100^{* *}$ \\
\hline
\end{tabular}

Note: Decimal points are omitted. Correlations comparing auditory and visual SS are above diagonal, those comparing visual and auditory SS below diagonal.

" $p<.05$

$* * p<.01$

exhibited a simplex-like pattern (cf. Guttman \& Guttman, 1965), i.e., the correlations between adjacent age groups are generally greater than those between widely spaced age groups. In addition, age-correlations of mean preferences were computed across sensory modalities. These cross-modality age-correlations are summarized in Table 2. Over-all both age-correlation matrices across sensory modalities are again similar to a simplex-pattern supporting the notion of a similar age trend in preferences for visual and auditory stimulus sequences. Thus, the cross-modality correlations within the same age levels are all significant at the $1 \%$ level and are generally as high as the test-retest correlations within sensory modalities. However, as a function of increasing age disparity, the correlations between age groups approach zero some reaching negative, though not significant values.

This consistent age trend towards preference for slower sequences both within auditory and visual stimulus material makes further studies along these lines quite appealing. It is not yet clear by which psychological dimensions (e.g., novelty, variability, complexity, meaningfulness) the different stimulus sequences may be characterized. However. one is tempted to relate the present pattern of age-related preferences to findings by Munsinger \& Kessen (1966) who reported a decreasing preference for figures of high variability with increasing age. In addition, by application of a multivariate strategy it seems feasible to explore the construct validity of the present findings within both a psychological as well as a physiological (e.g., heart-rate, EEG, metabolism) framework.

However, also with regard to Munsinger and Kessen's results, one should recognize that the straightforward interpretation of the obtained age differences as age effects is not yet fully warranted since generational differences (Schaie, 1965; Baltes, 1968) between the cross-sectional samples may be involved as well. Therefore, the present cross-sectional study will have to be followed up by adequate sequential strategies.

\section{REFERENCES}

BALTES, P. B. Longitudinal and cross-sectional sequences in the study of age and generation effects. Hum. Develpm., 1968, $11,145-171$.

BERLYNE, D. E. Conflict, arousal and curiosity. New York: McGraw-Hill, 1960.

BOESCH, E. E., BALTES, P. B., \& SCHMIDT, L. R. Preference for different auditory stimulus sequences in various age groups. Psychon. Sci. 1968, 10, 205-206.

FISKE, D. W., \& MADDI, S. R. Functions of varied experience. Homewood: Dorsey Press, 1961

GUTTMAN, R., \& GUTTMAN, L. A new approach to the analysis of growth patterns: the simplex structure of intercorrelations of measurements. Growth, 1965, 29, 219-232.

LEUBA, C. Relation of stimulation intensities to learning and development Psychol Rep., 1962, 11, 55-65.

MUNSINGER, H., \& KESSEN, W. Stimulus variability and cognitive change. Psychol Rev., 1966, 73, 164178.

SCHAIE, K. W. A general model for the study of developmental problems. Psychol. Bull., 1965, 64, 92-107.

\section{NOTES}

1. The authors owe thanks to R. Haas, C. D. Kaulin, H. Kraemer, Ursula Mainberger, Christine Schumann, and $H$. Zayer for their valuable assistance in data collection and data analysis, and to C. D. Corman. L. R. Goulet, and J. R. Nesselroade for a critical reading of the manuscript.

2. Data were collected while the first author was at the Universitaet des Saarlandes, Germany. 